\section{Avaliação dos cuidados de saúde: percepções de mulheres imigrantes em Portugal}

\section{Health care assessment: the perceptions of female immigrants in Portugal}

Sónia Dias 1

Ana Gama 2

Rosário Horta 3

1,2 Instituto de Higiene e Medicina Tropical. Universidade Nova de Lisboa. Rua da Junqueira, $\mathrm{n}^{\circ}$ 100. Lisboa, Portugal. 1349-008. E-mail: sfdias@ihmt.unl.pt

${ }^{3}$ Ministério da Saúde de Portugal. Lisboa, Portugal.

\begin{abstract}
Objectives: to describe the health care seeking behavior and perceptions relating to the use of health services of women immigrants residing in Portugal.

Methods: in 2009 a cross-sectional study was carried out in the metropolitan area of Lisbon, in which a questionnaire was applied to a sample of 703 women immigrants. The questionnaire included items relating to social and demographic characteristics and the health care seeking behavior and perceptions of the participants regarding the use of services. A descriptive analysis of the variables under study was carried out.

Results: most participants (82.4\%) reported that they had already used the National Health Service (SNS), although this response was more frequent among Africans (94.5\%) and East Europeans (87.2\%) than among Brazilians (66.8\%). Around $60 \%$ of respondents reported that they were satisfied or highly satisfied with the service, with the Africans responding in this manner more frequently (77\%) than the Brazilians (49.7\%) and East Europeans (43.4\%). The groups of participants point to various obstacles to the use of health services, including issues related their status as immigrants, interaction with health professionals and the services provided.

Conclusions: differences in the perceptions of immigrant groups regarding health services and professionals reflect the importance of developing evaluative practices that identify where intervention is needed. Knowing this would make it possible to better adapt the health care provided to specific immigrant groups.
\end{abstract}

Key words Evaluation, Women, Emigrants and immigrants, Health services, Personal satisfaction

\section{Resumo}

Objetivos: descrever práticas relacionadas com a procura de cuidados de saúde e percepções relativamente a aspectos da utilização dos serviços de saúde por parte de uma população de mulheres imigrantes a residir em Portugal.

Métodos: em 2009 foi realizado um estudo transversal na área metropolitana de Lisboa onde foi aplicado um inquérito por questionário a uma amostra de 703 mulheres imigrantes. O questionário incluiu itens para caracterização sóciodemográfica e sobre práticas relacionadas com a procura de cuidados de saúde e percepções das participantes quanto à utilização dos serviços. Realizou-se uma análise descritiva das variáveis em estudo.

Resultados: a grande maioria das participantes (82.4\%) reporta já ter utilizado o Serviço Nacional de Saúde (SNS), mais frequentemente as africanas (94,5\%) e do Leste Europeu (87,2\%) do que as brasileiras (66.8\%). Cerca de $60 \%$ das inquiridas revela estar satisfeita ou muito satisfeita com os serviços, mais frequentemente as africanas (77\%) do que as brasileiras (49,7\%) e do Leste Europeu $(43,4 \%)$. Os grupos de participantes apontam diferentes obstáculos para a utilização dos serviços de saúde, entre os quais aspectos relacionados com a condição de ser imigrante, a interação com os profissionais de saúde e os serviços.

Conclusões: as diferenças nas percepções dos grupos de imigrantes sobre os serviços e profissionais refletem a importância de desenvolver práticas avaliativas que identifiquem necessidades de intervenção. Tal conhecimento pode contribuir para melhor adequar a prestação de cuidados de saúde às especificidades dos grupos de imigrantes.

Palavras-chave Avaliação, Mulheres, Emigrantes e imigrantes, Serviços de saúde, Satisfação pessoal 


\section{Introdução}

O fenômeno crescente e global da migração internacional e feminina, e as diferenças nas necessidades em saúde dos vários grupos da população são atualmente considerados desafios para a política e sistemas de saúde dos países que procuram alcançar equidade. ${ }^{1-3} \mathrm{Na}$ verdade, uma das principais metas das políticas de saúde em diversos países tem sido a de promover essa equidade. Contudo, de um modo geral, a literatura aponta para desigualdades que tendem a prejudicar os grupos sociais mais desfavorecidos. ${ }^{4}$ Essa situação pode refletir possíveis desigualdades no acesso aos cuidados de saúde. 5 De fato, também de acordo com algumas investigações realizadas em Portugal, a associação positiva entre imigração e vulnerabilidade parece estar relacionada com um reduzido acesso e utilização dos serviços de saúde. 6-8 Esta questão torna-se especialmente relevante na medida em que tem sido amplamente reconhecido que as iniquidades no acesso aos serviços tendem também a penalizar os grupos mais vulneráveis social e economicamente, como os grupos de mulheres imigrantes, mantendo grande parte destas populações numa situação de maior vulnerabilidade. ${ }^{4}$ Assim, no sentido de reduzir essas iniquidades é cada vez mais reforçada a promoção de cuidados de saúde universalmente acessíveis e de qualidade. 9

$\mathrm{O}$ acesso e utilização dos serviços assumem um princípio básico para promover um elevado nível de saúde das populações. O estado de saúde, por sua vez, condicionado pela interdependência dos seus diversos determinantes, tem forte impacto no processo de uma boa integração do imigrante na sociedade de acolhimento. 10

No estado atual do conhecimento é importante adotar uma abordagem integrada da migração e um modelo social de saúde positiva em que os sistemas de saúde sejam capazes de responder de forma adequada aos desafios subjacentes à diversidade cultural. Neste sentido é fundamental conhecer as especificidades dos diferentes grupos populacionais e os seus contextos socioeconômicos e culturais, incluindo as necessidades subjetivas, valores, sentimentos e desejos que influenciam as práticas de saúde das populações. ${ }^{11}$

Uma primeira estratégia no sentido da identificação de prioridades em saúde e da integração dos valores e princípios socialmente partilhados tem sido o diagnóstico de necessidades no nível mais próximo da comunidade, através da participação dos atores envolvidos na produção dessas práticas.4,12 Esta prioridade tem sido reforçada particularmente pelo fato de persistir uma lacuna de estudos de avaliação da perspectiva das comunidades imigrantes no que diz respeito à saúde, ${ }^{4}$ sendo considerado fundamental conhecer as percepções sobre cuidados de saúde de comunidades com especificidades socioeconômicas e culturais. A avaliação destes aspectos é uma importante ferramenta para a produção de conhecimento que permita identificar necessidades e prioridades de intervenção, auxiliando na tomada de decisão e no estabelecimento de políticas e estratégias de saúde integradoras e sustentadas, que tenham efeitos reais na promoção e proteção da saúde destas comunidades, em particular no grupo das mulheres.

Por outro lado, a avaliação da perspectiva destes grupos sobre cuidados recebidos pode contribuir para avaliações futuras, servindo de estudo de linha de base para recolher e sistematizar um conjunto de dados essenciais que permitam qualificar intervenções, nomeadamente os processos implicados na produção de efeitos na melhoria das condições de saúde e do acesso e utilização dos serviços. A presente pesquisa pretende ser um contributo nesta área. Assim procurou-se descrever práticas relacionadas com a procura de cuidados de saúde e percepções relativamente a aspectos da utilização dos serviços por parte de uma população de mulheres imigrantes residentes em Portugal.

\section{Métodos}

Em 2009 foi realizado um estudo transversal na área metropolitana de Lisboa, localidade que tem atualmente a maior concentração de populações imigrantes do país. Um inquérito por questionário foi aplicado a 703 mulheres. No estudo foi desenvolvido um processo de amostragem por snowball. ${ }^{13}$ Neste processo estiveram envolvidas organizações não governamentais (ONGs) e associações de imigrantes das comunidades africana, brasileira e de países do Leste Europeu, que colaboraram com a equipe de investigação identificando e recrutando participantes. A estas participantes foi pedido, após responderem ao questionário, que identificassem e recrutassem elementos das suas comunidades, redes sociais e relacionais para participar no estudo.

O questionário incluiu itens para a caracterização sóciodemográfica das participantes, especificamente sobre idade, nível educacional, religião, situação profissional, situação administrativa e tempo de residência em Portugal. O instrumento de recolha de dados incluiu ainda questões sobre práticas relacionadas com a procura de cuidados de saúde e percepções quanto à utilização dos serviços.

$\mathrm{O}$ recolhimento de dados foi realizada por entre- 
vistadores treinados e oriundos das comunidades em estudo. O consentimento informado e voluntário das participantes foi obtido com garantia do anonimato e da confidencialidade dos dados. O estudo foi aprovado pelo Conselho de Ética do Instituto de Higiene e Medicina Tropical.

A informação coletada foi armazenada numa base de dados e processada no programa SPSS 17.0, realizando-se análise descritiva das variáveis.

\section{Resultados}

Do total de participantes, 36,4 \% $(\mathrm{n}=256)$ são oriundas de países africanos de língua oficial portuguesa (PALOP), 36,8 \% $(\mathrm{n}=259)$ do Brasil e $26,8 \%(\mathrm{n}=188)$ de países do Leste Europeu (Tabela 1). As participantes têm maioritariamente entre $25 \mathrm{e}$ 44 anos de idade. A maior parte das mulheres africanas apresenta nível básico de escolaridade $(\leq 4$ anos: $32,9 \%$ ), as brasileiras nível intermédio (10-12 anos: 44,6\%) e as do Leste Europeu nível superior ( $\geq 13$ anos: $75,3 \%$ ). A maioria das participantes africanas $(82,5 \%)$ e brasileiras $(51,8 \%)$ é católica e a generalidade das mulheres do Leste Europeu é ortodoxa $(84,1 \%)$.

\section{Tabela 1}

\begin{tabular}{|c|c|c|c|c|c|c|c|c|}
\hline \multirow[t]{2}{*}{ Características } & \multicolumn{2}{|c|}{ Total } & \multicolumn{2}{|c|}{ Africanas } & \multicolumn{2}{|c|}{ Brasileiras } & \multicolumn{2}{|c|}{ Leste Europeu } \\
\hline & $\mathrm{n}$ & $\%$ & $\mathrm{n}$ & $\%$ & $\mathrm{n}$ & $\%$ & $\mathrm{n}$ & $\%$ \\
\hline Idade (anos) & 702 & 100,0 & & & & & & \\
\hline $18-24$ & 109 & 15,5 & 54 & 21,2 & 41 & 15,8 & 109 & 15,5 \\
\hline $25-44$ & 428 & 61,0 & 121 & 47,5 & 184 & 71,0 & 428 & 61,0 \\
\hline $45-64$ & 145 & 20,7 & 61 & 23,9 & 34 & 13,1 & 145 & 20,7 \\
\hline $65-70$ & 20 & 2,8 & 19 & 7,5 & 0 & 0,0 & 20 & 2,8 \\
\hline Escolaridade (anos) & 687 & 100,0 & & & & & & \\
\hline$\leq 4$ & 93 & 13,5 & 84 & 32,9 & 9 & 3,5 & 0 & 0,0 \\
\hline $5-6$ & 67 & 9,8 & 37 & 14,5 & 29 & 11,2 & 1 & 0,6 \\
\hline $7-9$ & 87 & 12,7 & 59 & 23,1 & 22 & 8,5 & 6 & 3,4 \\
\hline $10-12$ & 205 & 29,8 & 54 & 21,2 & 115 & 44,6 & 36 & 20,7 \\
\hline$\geq 13$ & 235 & 34,2 & 54 & 8,2 & 83 & 32,2 & 131 & 75,3 \\
\hline Religião & 687 & 100,0 & & & & & & \\
\hline Católica & 357 & 52,0 & 208 & 82,5 & 131 & 51,8 & 18 & 9,9 \\
\hline Ortodoxa & 154 & 22,4 & 1 & 0,4 & 0 & 0,0 & 153 & 84,1 \\
\hline Protestante/Evangélica & 84 & 12,2 & 8 & 3,2 & 75 & 29,6 & 1 & 0,6 \\
\hline Sem religião & 39 & 5,7 & 17 & 6,7 & 20 & 7,9 & 2 & 1,1 \\
\hline Outra & 53 & 7,7 & 18 & 7,2 & 27 & 10,7 & 8 & 4,3 \\
\hline Situação profissional & 697 & 100,0 & & & & & & \\
\hline Empregado & 484 & 69,5 & 146 & 57,9 & 186 & 72,4 & 152 & 81,3 \\
\hline Desempregado & 111 & 16,0 & 58 & 23,0 & 34 & 13,3 & 19 & 10,2 \\
\hline Reformado & 17 & 2,4 & 17 & 6,8 & 0 & 0,0 & 0 & 0,0 \\
\hline Estudante & 50 & 7,2 & 21 & 8,3 & 24 & 9,3 & 5 & 2,7 \\
\hline Outra & 34 & 4,9 & 10 & 4,0 & 13 & 5,0 & 11 & 5,8 \\
\hline Situação administrativa & 689 & 100,0 & & & & & & \\
\hline Regular & 538 & 78,1 & 212 & 85,5 & 163 & 63,7 & 163 & 78,1 \\
\hline Irregular & 46 & 6,7 & 13 & 5,2 & 27 & 10,5 & 6 & 6,7 \\
\hline Processo de regularização & 105 & 15,2 & 23 & 9,3 & 66 & 25,8 & 16 & 15,2 \\
\hline Tempo de residência em Portugal (anos) & 672 & 100,0 & & & & & & \\
\hline$<3$ & 182 & 27,1 & 34 & 15,0 & 125 & 48,4 & 23 & 12,3 \\
\hline $3-5$ & 110 & 16,4 & 12 & 5,3 & 64 & 24,8 & 34 & 18,2 \\
\hline $5-10$ & 255 & 37,9 & 67 & 29,5 & 61 & 23,7 & 127 & 67,9 \\
\hline$>10$ & 125 & 18,6 & 114 & 50,2 & 8 & 3,1 & 3 & 1,6 \\
\hline Total & 703 & 100,0 & 256 & 36,4 & 259 & 36,8 & 188 & 26,8 \\
\hline
\end{tabular}


Do total de participantes, cerca de 78,0\% encontra-se em situação regular. As mulheres brasileiras reportam mais frequentemente estar em processo de regularização $(25,1 \%)$ ou em situação irregular (10,5\%). A maior parte das participantes africanas reside há mais tempo em Portugal ( $>10$ anos: 50,2\%) enquanto as brasileiras reportam menor tempo de residência no país $(<3$ anos: $48,4 \%$; 3-5 anos: $24,8 \%$ ). Cerca de $70,0 \%$ das participantes encontra-se empregada.

A grande maioria das participantes $(82,4 \%$, $\mathrm{n}=579$ ) reporta já ter utilizado o Serviço Nacional de Saúde (SNS), mais frequentemente as africanas $(94,5 \%, n=242)$ e do Leste Europeu $(87,2 \%, n=164)$ do que as brasileiras $(66,8 \%, \mathrm{n}=173)(p<0,001)$. Mais especificamente, $76,5 \%$ das inquiridas $(n=537)$ já utilizaram o centro de saúde (africanas: 91,4\%, $\mathrm{n}=233$; do Leste Europeu: 83,5\%, $\mathrm{n}=157$; brasileiras: $56,8 \%, \mathrm{n}=147)$ e cerca de $66,0 \%(\mathrm{n}=465)$ utilizou o hospital (africanas: 85,5\%, $\mathrm{n}=219$; do Leste Europeu: $62,9 \%, \mathrm{n}=117$; brasileiras: $49,8 \%, \mathrm{n}=129$ ).

Entre as participantes que referem nunca ter utilizado o SNS, as principais razões apontadas são não sentirem necessidade $(71,9 \%)$, o desconheci- mento dos procedimentos de utilização dos serviços de saúde $(26,0 \%)$ e da sua localização $(20,3 \%)$, e a percepção de mau atendimento aos imigrantes nestes serviços $(23,3 \%)$.

Quanto aos principais motivos para utilizar os serviços de saúde, as participantes africanas referem consultas de acompanhamento ou de rotina $(77,4 \%)$, realização de exames complementares de diagnóstico $(61,6 \%)$, consultas de saúde infantil e vacinação $(63,1 \%)$ e consultas de planejamento familiar e saúde materna $(56,2 \%)$. As mulheres brasileiras apontam mais frequentemente a utilização dos serviços de saúde em situações de urgência ou doença aguda (73,8\%), para consultas de acompanhamento ou de rotina $(65,9 \%)$ e para realização de exames complementares de diagnóstico (56,5\%), e as participantes do Leste Europeu referem utilizar os serviços em situações de urgência ou doença aguda $(77,6 \%)$ e para solicitar baixa médica $(61,0 \%)$.

Cerca de $60,0 \%$ das inquiridas revela estar satisfeita ou muito satisfeita com os serviços de saúde, mais frequentemente as africanas $(77,0 \%)$ do que as brasileiras $(49,7 \%)$ e as do Leste Europeu $(43,4 \%)$ (Tabela 2).

Nível de satisfação de mulheres imigrantes com os serviços de saúde. Lisboa, Portugal, 2009.

\begin{tabular}{|c|c|c|c|c|c|c|c|c|c|}
\hline & \multicolumn{2}{|c|}{ Total } & \multicolumn{2}{|c|}{ Africanas } & \multicolumn{2}{|c|}{ Brasileiras } & \multicolumn{2}{|c|}{ Leste Europeu } & \multirow[t]{2}{*}{$p$} \\
\hline & $\mathrm{n}$ & $\%$ & $\mathrm{n}$ & $\%$ & $\mathrm{n}$ & $\%$ & $\mathrm{n}$ & $\%$ & \\
\hline Insatisfeito/muito insatisfeito & 78 & 13,4 & 21 & 8,6 & 29 & 16,8 & 28 & 16,9 & $<0,001$ \\
\hline Nem satisfeito/nem insatisfeito & 159 & 27,3 & 35 & 14,3 & 58 & 33,5 & 66 & 39,8 & \\
\hline Satisfeito/muito satisfeito & 346 & 59,3 & 188 & 77,0 & 86 & 49,7 & 72 & 43,4 & \\
\hline
\end{tabular}

As participantes foram questionadas sobre quais os fatores que consideram ser um obstáculo para a utilização dos serviços. A maioria aponta o estar sozinha e há pouco tempo em Portugal (79,3\%), a falta de conhecimentos sobre direito à saúde e modo de funcionamento dos serviços $(78,9 \%)$ e os procedimentos burocráticos complexos de acesso aos serviços de saúde $(76,1 \%)$ (Tabela 3 ). A falta de recursos econômicos para suportar os custos dos serviços é referida como uma dificuldade pela maioria das participantes $(75,8 \%)$, mais frequentemente pelas africanas $(89,1 \%)$ do que pelas do Leste Europeu $(73,5 \%)$ e brasileiras $(64,6 \%)$. Cerca de metade $(49,3 \%)$ refere ainda como obstáculo aspectos relacionados com a expressão de sintomas e reconhecimento da doença, $48,3 \%$ refere a descon- fiança quanto aos profissionais de saúde e $47,7 \%$ aponta a ausência de tradutores/intérpretes nos serviços.

A maioria das participantes avalia positivamente o atendimento dos profissionais dos serviços de saúde, mais frequentemente o dos enfermeiros $(72,4 \%)$, seguido dos médicos $(68,2 \%)$ e dos administrativos $(51,7 \%)$. Uma maior proporção de mulheres brasileiras e do Leste Europeu avalia de forma negativa o atendimento dos administrativos ( $18,9 \%$ e $17,9 \%$, respectivamente) e dos médicos (13,8\% e $8,5 \%$, respectivamente), em comparação com as mulheres africanas (administrativos: 9,8\%, $p<0,001$; médicos: 4,1\%, $p<0,001)$.

Os administrativos são considerados como os profissionais que mais discriminam $(82,4 \%)$, os 


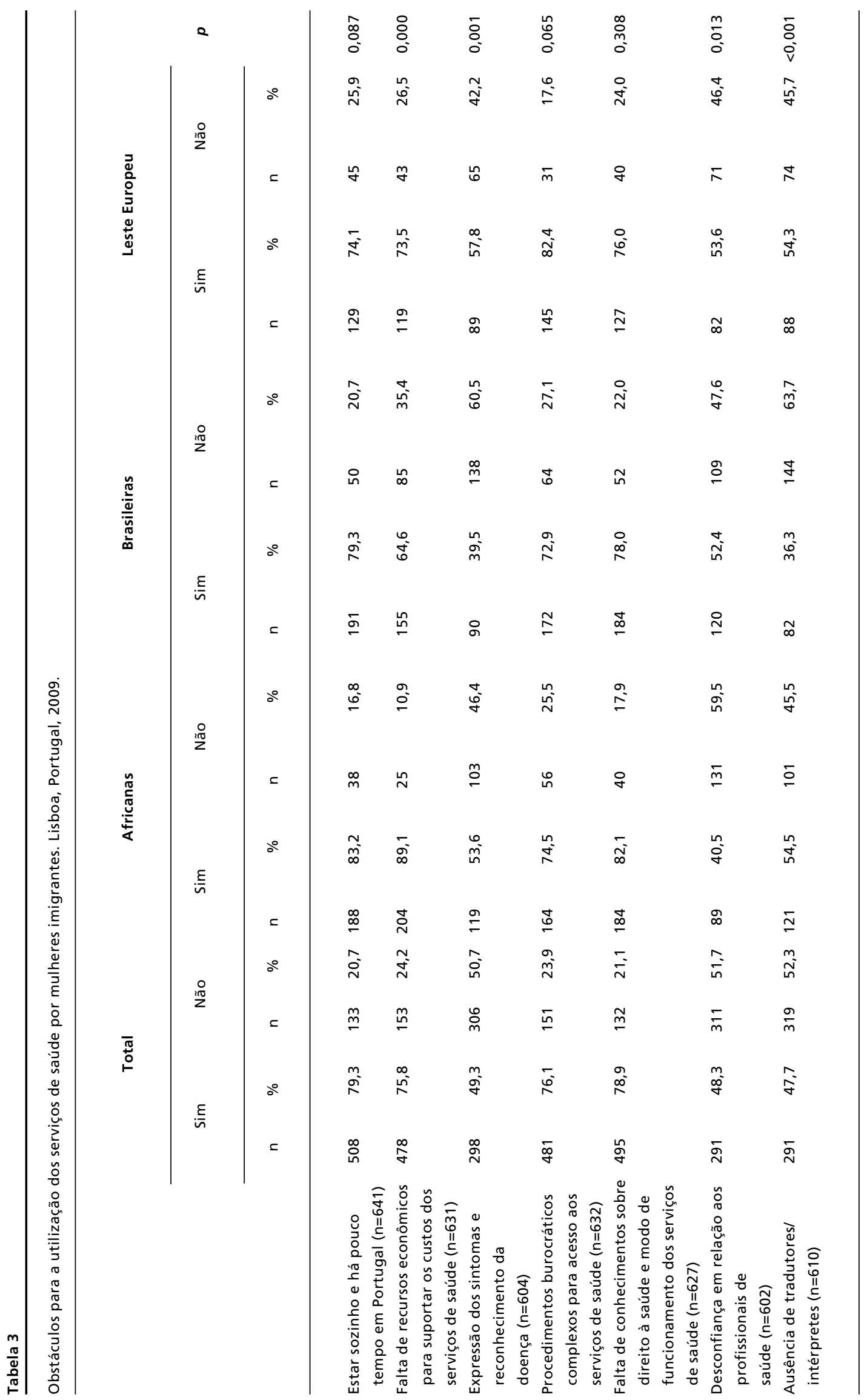


menos compreensivos quanto aos problemas dos imigrantes $(73,7 \%)$, os que têm mais dificuldades em compreendê-los $(73,2 \%)$ e os menos atenciosos $(69,1 \%)$. Os médicos são considerados pela maioria das inquiridas $(60,1 \%)$ como o grupo profissional que mais ouve os problemas dos pacientes. Esta percepção é significativamente mais frequente entre as participantes africanas $(70,9 \%)$ do que entre as do Leste Europeu $(55,5 \%)$ e as brasileiras $(51,4 \%)$.

\section{Discussão}

A iniciativa de avaliar na área da saúde passa pela necessidade de preencher lacunas no conhecimento, analisando e julgando ações e atividades desenvolvidas para conhecer e modificar uma situação, mediante a tomada de novas decisões. 14,15 De acordo com vários modelos do uso da avaliação descritos na literatura, a avaliação pode ser usada numa perspectiva instrumental para a tomada de decisões ou introdução de alterações nos programas, numa perspectiva política para a criação de novas propostas ou modelos de programas e legitimação de decisões, e numa perspectiva conceitual para produção de conhecimento. 16,17

A nível internacional tem sido notório o interesse em incluir na avaliação, e mais especificamente do sistema de saúde, a perspectiva do usuário.18,19 Neste contexto, "a incorporação do usuário na avaliação tem sido valorizada não apenas por constituirse um indicador sensível da qualidade do serviço prestado, mas por estar potencialmente relacionada à maior adequação no uso do serviço". 20 De fato, o conhecimento das percepções dos usuários sobre a acessibilidade, qualidade do atendimento e resolutividade dos serviços de saúde e do seu grau de satisfação têm sido considerados importantes indicadores da qualidade desses serviços e para o seu melhoramento. ${ }^{19,20} \mathrm{Na}$ perspectiva de avaliação do desempenho do sistema de saúde e da efectividade da política setorial também tem sido considerada relevante a inclusão da mensuração da satisfação dos usuários nos instrumentos de avaliação. ${ }^{21}$

$\mathrm{O}$ estudo apresentado permitiu conhecer as práticas relacionadas com a utilização dos serviços de saúde por parte de uma população de mulheres imigrantes residentes em Portugal e as percepções delas sobre esses serviços. As informações recolhidas nesta prática avaliativa podem ser usadas como indicadores da qualidade de diversos aspectos dos serviços como a acessibilidade, a qualidade do atendimento dos profissionais e, na globalidade, ao nível da política de saúde.

Os resultados revelam diferenças nos grupos de mulheres imigrantes relativamente a práticas relacionadas com a procura de cuidados de saúde. De uma forma geral, o grupo de africanas reporta maior utilização dos serviços de saúde para obter diversos tipos de cuidados, quer seja para consultas de rotina, de planejamento familiar, de saúde materno-infantil e vacinação, quer seja para apoio social. Por outro lado, uma menor utilização dos serviços foi reportada pelas mulheres brasileiras e do Leste Europeu. Para estas participantes, o principal motivo da utilização dos serviços foi o fato de se encontrarem em situação de urgência ou doença aguda. Vários fatores têm sido apontados para explicar diferenças nos padrões de utilização dos serviços de saúde por parte das populações imigrantes, tais como o tempo de residência no país de acolhimento, 22 a situação administrativa do imigrante, 6,23 a situação socioeconômica 24 ou fatores culturais. $25,26 \mathrm{O}$ conhecimento sobre os diferentes motivos de utilização dos serviços de saúde por parte das mulheres imigrantes e os fatores associados pode contribuir para uma melhor adequação desses serviços às especificidades e necessidades dos vários grupos dessa população.

Apesar de os resultados indicarem um panorama positivo quanto à utilização do SNS por parte das mulheres imigrantes, uma importante proporção nunca utilizou estes serviços, o que pode representar oportunidades perdidas de atuação ao nível da promoção da saúde e prevenção da doença. Os principais motivos da não utilização dos serviços de saúde apontados pelas participantes foram o desconhecimento dos procedimentos para a sua utilização e da localização, e a percepção de que os imigrantes são mal atendidos. Estes resultados vão ao encontro dos de outros estudos 6,27 e são importantes pois podem contribuir para o delineamento de estratégias eficazes para a redução de barreiras no acesso e utilização dos serviços de saúde.

A generalidade das participantes apontou fatores relacionados com a condição de ser imigrante e os serviços de saúde como obstáculos para a sua utilização. Outros estudos desenvolvidos em Portugal com populações imigrantes têm demonstrado resultados semelhantes. ${ }^{6-8}$ Mais recentemente foi desenvolvido um estudo qualitativo com a realização de grupos focais com mulheres imigrantes oriundas de PALOP e do Brasil onde, segundo os discursos registrados, as participantes consideraram existir barreiras administrativas, estruturais, organizativas, socioeconômicas e culturais no acesso e utilização dos serviços de saúde. 3

No presente estudo verificaram-se diferenças nas percepções sobre os obstáculos para a utilização dos serviços de saúde de acordo com os grupos de 
origem. As mulheres africanas referiram mais frequentemente a falta de recursos econômicos, o que pode em parte ser explicado pelo fato deste grupo apresentar pior situação socioeconômica (em comparação com os outros grupos, uma maior proporção de africanas encontra-se desempregada e tem nível de escolaridade inferior).

As participantes africanas e do Leste Europeu referiram mais frequentemente dificuldades relacionadas com a expressão dos sintomas e reconhecimento da doença. Estes resultados apontam no sentido de que os fatores culturais condicionam as percepções e atitudes sobre saúde e doença que influenciam a forma como os sintomas são reconhecidos e têm efeitos ao nível da utilização dos serviços de saúde. 25 Torna-se assim importante conhecer as especificidades culturais das populações imigrantes de forma a adequar a prestação de cuidados aos diferentes grupos. Por outro lado, os resultados parecem indicar que as questões linguísticas são também importantes fatores da utilização dos serviços pelos imigrantes. De fato, as mulheres africanas e do Leste Europeu referiram como obstáculos a ausência de tradutores ou intérpretes nos serviços, o que reforça a necessidade de desenvolver iniciativas e estratégias que permitam ultrapassar as diferenças linguísticas nos serviços de saúde. Tais iniciativas podem incluir a disponibilização de informação escrita em várias línguas, a formação de profissionais de saúde para o desenvolvimento de competências linguísticas ou a criação de serviços de tradução. Por outro lado, as mulheres brasileiras e do Leste Europeu referiram mais frequentemente como obstáculo a desconfiança dos imigrantes em relação aos profissionais de saúde, o que realça a importância de desenvolver iniciativas com vista à melhoria da relação profissional/paciente, bem como aprofundar o conhecimento sobre as percepções das mulheres imigrantes sobre estes profissionais.

De forma geral, as mulheres africanas revelaram maior grau de satisfação com os serviços do que o grupo das brasileiras e do Leste Europeu. Vários estudos têm indicado que a etnicidade, cultura e país de origem estão frequentemente associados à percepção de qualidade e satisfação com os serviços de saúde do país de acolhimento.27,28 Alguns autores têm referido que o grau de satisfação das populações imigrantes com o sistema de saúde é em parte influenciado pelas suas expectativas quanto aos cuidados de saúde e experiências anteriores no país de origem.29,30 Assim, é possível admitir que populações oriundas de regiões com falta de estruturas de cuidados de saúde podem percepcionar os serviços em Portugal como de maior qualidade, enquanto que populações oriundas de regiões com um sistema de saúde eficiente podem percepcionar os serviços em Portugal como menos competentes.6,29

$\mathrm{Na}$ generalidade, as participantes avaliaram de forma positiva o atendimento dos profissionais de saúde. No entanto, em comparação com as mulheres africanas, uma maior proporção de mulheres brasileiras e do Leste Europeu avaliaram de forma negativa o atendimento destes profissionais. Para a maioria das participantes, os administrativos foram considerados como os profissionais menos atenciosos, os menos compreensivos quanto aos problemas dos imigrantes, os que têm mais dificuldades em compreendê-los e os que mais discriminam, percepções que também foram expressas no estudo qualitativo de Dias e Rocha. ${ }^{3}$ Nas discussões dos grupos focais foram ainda descritos episódios de discriminação, seja racial seja sexual, por parte dos profissionais de saúde.

Tem sido apontada por diversos autores a pouca preparação que alguns profissionais de saúde apresentam para lidar com a diversidade cultural que caracteriza atualmente a população usuária dos serviços. 26,27 Neste contexto, será importante desenvolver um trabalho com os profissionais de saúde ao nível da promoção de competências culturais para que estejam sensibilizados e capacitados para lidar com as diferentes populações. Neste sentido, devem ser implementados programas de formação e capacitação continuada junto dos profissionais de saúde, adequados ao perfil funcional de cada grupo profissional e às suas necessidades específicas, com vista a um maior conhecimento das populações imigrantes e dos diferentes fatores que têm impacto na sua tomada de decisão em questões relacionadas com a saúde.

A avaliação do atendimento nos serviços de saúde tem sido considerada importante para delinear estratégias de intervenção no sentido de uma maior qualidade dos serviços e equidade em saúde. De fato, tem sido reconhecido que a simples disponibilidade dos serviços não é suficiente para a sua utilização. Outros fatores têm uma grande influência como seja a capacidade dos serviços e profissionais de garantir um atendimento adequado às populações usuárias. 15 As diferenças nas percepções dos vários grupos de imigrantes sobre os serviços e profissionais de saúde que se verificaram neste estudo refletem a importância de desenvolver práticas avaliativas identificando necessidades de intervenção para melhor adequar a prestação de cuidados às especificidades dos grupos.

Os resultados desta prática avaliativa reforçam a 
pertinência de melhorar a acessibilidade dos serviços de saúde ao nível da relação entre profissionais e usuários, bem como ao nível da sua estrutura e procedimentos. Contudo, o nosso estudo mostra que os esforços que têm sido desenvolvidos em Portugal na implementação de políticas e estratégias de ação para a promoção do acesso e utilização dos serviços nas populações imigrantes têm tido resultados positivos. 3,6

$\mathrm{O}$ presente estudo coloca-se também como pesquisa participativa envolvendo as comunidades e de certa forma contribuindo para a sua sensibilização na área da saúde, o que representa uma mais valia para o empowerment das comunidades imigrantes.

Em conclusão, esse estudo vem reforçar a importância e a necessidade de continuar a desen-

\section{Referências}

1. Carballo M, Divino JJ, Zeric D. Migration and health in the European Union. Trop Med Int Health. 1998; 3: 936-44.

2. Dias S, Gonçalves A. Migração e saúde. Migrações. 2007; 1: $15-26$.

3. Dias SF, Rocha CF. Saúde sexual e reprodutiva de mulheres imigrantes africanas e brasileiras: um estudo qualitativo. Lisboa: ACIDI; 2009.

4. Carvalho AI, Bodstein RC, Hartz Z, Matida AH. Concepts and approaches in the evaluation of health promotion. Ciênc Saúde Coletiva. 2004; 9: 521-9.

5. Stronks K, Ravelli C, Reijneveld A. Immigrants in the Netherlands: equal access for equal needs? J Epidemiol Community Health. 2001; 55: 701-7.

6. Dias SF, Severo M, Barros H. Determinants of health care utilization by immigrants in Portugal. BMC Health Serv Res. 2008; 8: 207.

7. Gonçalves A, Dias S, Luck M, Fernandes J, Cabral J. Acesso aos cuidados de saúde de comunidades migrantes. Rev Port Saude Publica. 2003; 21: 55-64.

8. Machado MC, Santana P, Carreiro M, Nogueira H, Barroso M, Dias A. Iguais ou diferentes? Cuidados de saúde materno-infantil a uma população de imigrantes. Lisboa: Bial; 2006.

9. Campos CEA. Estratégias de avaliação e melhoria contínua da qualidade no contexto da atenção primária à saúde. Rev Bras Saúde Matern Infant. 2005; 5 (Supl 1): S63-9.

10. Whitehead M, Dahlgren G. Concepts and principles for tackling social inequities in health: Levelling up Part 1. Copenhagen: WHO; 2006.

11. Bosi MLM, Uchimura KY. Avaliação da qualidade ou avaliação qualitativa do cuidado de saúde. Rev Saúde Pública. 2007; 41: 150-3.

12. Escorel S. Os dilemas da equidade em saúde: aspectos conceituais. Brasília, DF: OPAS; 2001. [acesso em: 10 Mar 2010]. Disponível em: http://www.opas.org.br/servico/ Arquivos/Sala3310.pdf volver práticas avaliativas na área da saúde, com vista a subsidiar o desenvolvimento e implantação de estratégias inovadoras de promoção da saúde das populações imigrantes.

\section{Agradecimentos}

Este estudo foi financiado pela Fundação para a Ciência e a Tecnologia (IME/SAUESA/81760/ 2006). Os autores agradecem a todas as participantes do estudo. Os autores agradecem ainda aos membros da equipe de projeto, especialmente à Maria Cortes, António Carlos da Silva, Helena Cargaleiro, Mário Carreira, Violeta Alarcão e Miguel Lemos.

13. Vogt WP. Dictionary of statistics and methodology: a nontechnical guide for the social sciences. 3 ed. London: Sage; 2005.

14. Vieira-da-Silva LM. Conceitos, abordagens e estratégias para a avaliação em saúde. In: Hartz ZMA, Vieira-da-Silva LM, organizadores. Avaliação em saúde: dos modelos teóricos à prática na avaliação de programas e sistemas de saúde. Salvador: EDUFBA; Rio de Janeiro: Editora FIOCRUZ; 2005. p. 15-39.

15. Koffman MD, Bonadio IC. Avaliação da atenção pré-natal em uma instituição filantrópica da cidade de São Paulo. Rev Bras Saúde Matern Infant. 2005; 5 (Supl.1): S23-32.

16. Weiss CH, Murphy-Graham E, Birkeland S. An alternate route to policy influence: how evaluations affect D.A.R.E. Am J Eval. 2005; 26: 12-30

17. Hartz ZMA, Santos EM, Matida AH. Promovendo e analisando o uso e a influência das pesquisas avaliativas. In: Hartz ZMA, Felisberto E, Vieira-da-Silva LM, orgs. Metaavaliação da atenção básica à saúde: teoria e prática. Rio de Janeiro: Editora FIOCRUZ; 2008. p. 325-40.

18. Trad LAB, Bastos ACS, Santana EM, Nunes MO. Estudo etnográfico da satisfação do usuário do Programa de Saúde da Família (PSF) na Bahia. Ciênc Saúde Coletiva. 2002; 7: $581-9$.

19. Massote AW, Lima AMD, van Stralen CJ, Álvares J, Fleury-Teixeira P, Belisário SA, van Stralen TBS. A contribuição de estudos de percepção da população usuária na avaliação da atenção básica. In: Hartz ZMA, Felisberto E, Vieira-da-Silva LM, organizadores. Meta-avaliação da atenção básica à saúde: teoria e prática. Rio de Janeiro: Editora FIOCRUZ; 2008. p. 237-52.

20. Esperidião M, Trad L. Avaliação de satisfação de usuários. Ciênc Saúde Coletiva. 2005; 10 (supl.): 303-12.

21. Szwarcwald C, Mendonça M, Andrade C. Indicadores de atenção básica em quatro municípios do estado do Rio de Janeiro, 2005: resultados de inquérito domiciliar de base populacional. Ciênc Saúde Coletiva. 2006; 11: 643-55. 
22. Leduc N, Proulx M. Patterns of health services utilization by recent immigrants. J Immigr Health. 2004; 6: 15-27.

23. Torres-Cantero AM, Miguel AG, Gallardo C, Ippolito S Health care provision for illegal migrants: may health policy make a difference? Eur J Public Health. 2007; 17: 483-5.

24. Jones EM, Cason CL, Bond ML. Access to preventive health care: is method of payment a barrier for immigrant Hispanic women? Womens Health Issues. 2002; 12: 12937.

25. Anderson LM, Scromshaw SC, Fullilove MT, Fielding JE, Normand J, Task Force on Community Preventive Services. Culturally competent healthcare systems: a systematic review. Am J Prev Med. 2003; 24 (Supl 3): 68-79.

26. Eshiett MU, Parry EH. Migrants and health: a cultural dilemma. Clin Med. 2003; 3: 229-31.

Recebido em 14 de abril de 2010

Versão final apresentada em 26 de junho de 2010

Aprovado em 9 de julho de 2010
27. Scheppers E, van Dongen E, Dekker J, Geertzen J, Dekker J. Potential barriers to the use of health services among ethnic minorities: a review. Fam Pract. 2006; 23: 325-48.

28. Adamson J, Ben-Shlomo Y, Chaturvedi N, Donovan J. Ethnicity, socio-economic position and gender - do they affect reported health-care seeking behaviour? Soc Sci Med. 2003; 57: 895-904.

29. Gurman TA, Becker D. Factors affecting Latina immigrants' perceptions of maternal health care: findings from a qualitative study. Health Care Women Int. 2008; 29: $507-$ 26

30. Sofaer S, Firminger K. Patient perceptions of the quality of health services. Annu Rev Public Health. 2005; 26: 513-9. 